\title{
How the Players Get Their Spots: A Study of Playstyle Emergence in Digital Games
}

\author{
Milan Jaćević \\ Institute for Visual Design \\ The Royal Danish Academy - Architecture, Design, Conservation \\ Copenhagen, Denmark \\ mija@kglakademi.dk
}

\begin{abstract}
How, when, and why do players settle into a particular playstyle when playing a new digital game? Though some aspects of these questions have been addressed in player research (e.g. through player typologies), we are still lacking comprehensive answers that adequately account for the roles of both the player and the game in the manifestation of playstyles. The qualitative study presented here is a middle-ground look into how playstyles emerge when players sit down to play a new digital game. It frames playstyles as an in-game function of the player's ludic habitus - their past experiences, knowledge, and attitudes. The study takes the form of a playtest with ten players, using a custom adventure game/hypertext fiction prototype developed in Twine. The prototype offered two modes of engagement - slower reading of poetic text, and faster-paced exploration and puzzle-solving. The study found that playstyles consolidate at specific moments of discovery (e.g. upon solving an early puzzle), when the player's ludic habitus contextually interprets game design cues and reacts with a player-preferred form of engagement.
\end{abstract}

Keywords-playstyle, engagement, habitus, subfields of practice, player studies, game design

\section{INTRODUCTION}

In both game and player studies, researchers have discussed what differentiates players from others in their manner of play of certain games - often referred to as their playstyle. Such research has elaborated on the connections between playstyle and, among others, topics such as gender (e.g. $[18,20])$, age (e.g. [26, 31]), queer identities (e.g. [29]) and ethnicities (e.g. [6]). The depth and variety of existing research show that playstyles touch upon many elements of player psychology, sociology, and game design. Despite this work, fundamental questions about playstyles remain unanswered. How do playstyles emerge during gameplay? What makes a player play a particular game in a particular fashion? These issues are indicative of a broader lack of understanding about the practical, moment-to-moment activity of gameplay, which calls for novel methodologies and research perspectives to properly address it.

The exploratory qualitative player study in this research paper seeks to further our understanding of how, why, and when players settle into certain playstyles understood in this context as characteristic forms of engagement with a digital game ${ }^{l}$. Where existing research has examined player or game factors which might contribute to certain playstyles, or created playstyle classifications or categorizations, the present study is a middle-ground approach, bridging player and game research; it looks at how forms of engagement emerge when players sit down to play a new digital game title. It examines player interaction with a custom digital game prototype, developed in Twine, which combines design elements from both adventure games and hypertext fiction. Part of a broader research project that aims to extend Bourdieusian practice theory to interactions with digital games, the study is distinguished from previous work in the field by its examination of multiple player and game design factors pertaining to playstyle emergence. It answers the questions about playstyle emergence by framing playstyles as influenced by the players' game-domain-related experiences, knowledge, and attitudes - their ludic habitus and their perception and understanding of the broader field of digital gaming, including the specialized subfields of practice contained therein and clustered around games of specific types or genres. In examining playstyles as they come into being, at specific moments of discovery during the act of gameplay, this research contributes a better understanding of the activity of gameplay as being founded on and characterized by the interplay between the psychological and sociological player attributes and different components of a game's design.

The paper is structured in four parts. The first is an overview of existing classificatory player and game research, which showcases current perspectives on different styles of play of digital games. This section also details the theoretical framework used in the present study - Bourdieusian practice theory, namely the concepts of habitus and field, as well as their game-specific manifestations (ludic habitus and gaming field/subfields of practice). The following section presents the methodology of the exploratory qualitative player study, including details on the design of the prototype used in the study. The third section contains the results of the study, as well as the discussion of its findings. The paper ends with concluding remarks on playstyle emergence derived from the study.

\section{Playstyles IN Player AND GAME Research}

In research, playstyles have generally been examined from one of two perspectives: one that focuses on players, and one that focuses on game design. Before explaining how the present research aims to bridge these two traditions, it is worth briefly presenting this previous work.

The idea of different styles of playing digital games has given rise to classificatory work on player typologies or taxonomies. In their metareview of this form of research, Hamari and Tuunanen found that existing player typologies have predominately been made on one of two bases: behavioral (i.e. based on players' in-game actions) and psychographic (i.e. based on players' psychosocial

${ }^{1}$ Understood as that subset of games which require some form of electronic computation for their operation. 
characteristics seen as pertinent to gaming) [17, p. 32]. Behavioral research has been conducted using forum post analyses [4], performance data generated during play of certain titles [15, 16], surveys [19], and/or interviews and focus groups [21]. On the other hand, psychographic player research has frequently looked into topics like player motivations, as part of either empirical [28, 34] or more theoretical studies [7]. Psychographic studies have also examined player preferences $[11,23,32]$, as well as the clustering of several psychological traits related to gaming, rather than just individual ones [5]. More recently, psychographic investigations have led to new theoretical frameworks regarding agency in digital games, and a more complex understanding of the emotional aspects of the gameplay experience [12].

The idea of different formats or styles of play experience can also be examined from the perspective of game artefacts, in the context of genres or game types rather than player types. The concept of playstyle has featured, explicitly or implicitly, in various genre classifications [33] and in game genre research in general $[1,3,27]$. In this form of research, playstyles figure as patterns of play behavior afforded by particular game design choices, in common to games of a particular kind or type. For example, according to Arsenault, game genres represent "the codified usage of particular mechanics and game design patterns to express a range of intended play-experiences" [3, p. 171, italics original]. From this standpoint, different genres of games afford different formats or types of play; consequently, these design affordances form the bases for different playstyles in a game.

The two research strands illustrated here, centered on players and games respectively, describe different influences on playstyles in digital games. However, all of these theories operate on very broad temporal frames, often framing playstyles as fixed properties of players, rather than examining how playstyles manifest and evolve during the act of playing specific titles. Addressing these concerns and expanding our understanding of playstyles - their formation and manifestation - calls for new research methods and designs, able to better examine and account for the complex confluence of player and game. The exploratory study described in this paper is an example of one such project, and has as its theoretical basis the work of Pierre Bourdieu, in particular his concepts of habitus and field.

\section{A. Ludic habitus and subfields of practice}

Since both player- and game-related factors seem to be relevant for discussing playstyles, we need to employ a perspective that encompasses both in order to investigate how playstyles emerge. For this reason, this research turns to Pierre Bourdieu's work on practice. His practice theory can be viewed as a constructivist form of structuralism [8, p. 14; 9 , p. 14], which reintroduces the figure of the agent into a network of relations with objective social positions in a particular domain. This middle-ground approach makes Bourdieu's work suitable for addressing the divide between player research and game research when approaching playstyles in games.

Two of Bourdieu's concepts, closely coupled in his understanding of all forms of human practice, are highly relevant within the context of the present research project. They are habitus and field.

Habitus is a system of dispositions - thoughts, beliefs, actions, etc. - which structures one's understanding of the cultural and practical field that produces it, and, in turn, acts as a generator of sensible, intelligible practices within said field. Habitus is the result of an agent's prolonged participation in activities within a given field of practice, which is populated by other agents, artefacts, and institutions, and which fosters certain norms and values at the expense of others. For example, a person's music habitus can be seen as comprising patterns of their activities of music production and consumption, tastes and attribution of value for particular musical genres, performers, or institutions, and forms and degrees of cultural involvement within the field of music, amongst other things. In other words, one's music habitus is the rich, detailed description at the heart of the fundamental questions of how one relates to, understands, and engages with (the field of) music.

The concepts of habitus and field be adapted for the domain of games. The cultural and practical domain in which a player builds their game-related or ludic habitus - i.e., where one becomes a player - is the ludic field, encompassing not only game artefacts, but also agents and institutions which operate in their creation, distribution, and valuation developers, publishers, reviewers, YouTubers, etc. Furthermore, genre groupings of digital games can be seen as distinct subfields in their own right - as discrete subfields of practice. Practical and cultural experience with these subfields - for example, with first-person shooter (FPS) or grand strategy subfields - serves to specialize and differentiate one's ludic habitus from that of another player.

Though both habitus and field have previously been used in research on digital games and players (see e.g. [2, 13, $14,22,24,35])$, they have not been the subject of in-depth empirical investigations on playstyles and play behavior. The present study aims to rectify that. Within the context of this paper, habitus will be used as a conceptual tool for discussing playstyles and player-related factors which influence their emergence, ultimately being framed (in accordance with Bourdieu's view) as a generator of characteristic play practices. The following section of the paper details the exploratory study, beginning with a description of the custom prototype created for investigating playstyle emergence in digital games.

\section{EXPLORATORY STUDY}

\section{A. Inglenook}

The game prototype, titled Inglenook, was developed for the purposes of examining how, why, and when playstyles emerge during digital gameplay. Its visual design was inspired by concrete poetry - a style of poetic writing which features unorthodox typographical arrangements of textual elements. The design goal which guided the prototype development was to create a twodimensional textual space for players to explore. In Inglenook, this space ended up taking the form of a house composed of textual elements, arranged so as to suggest shapes of certain objects (as seen in Figures 1 and 2). 
Inglenook was developed in Twine, a software tool primarily used to create hypertext fiction in the form of interlinked HTML pages referred to as passages. Each passage in the game consists of strings of words which together construct the physical space of the game, and a vertical text string - the word YOU - which is positioned at a particular location on the screen. Several of these passages, each with a different placement of the vertical YOU, collectively comprise a single navigable physical space in the game (e.g. a living room with lights, windows, and furniture). The player navigates through the game by moving between these passages, using the arrow keys on a keyboard ${ }^{2}$, and interacts with objects in the virtual environment by using the Space bar. Because of the change in position of the vertical YOU, the movement between passages registers as traversal of a $2 \mathrm{D}$ space. Figures 1 and 2 are meant to serve as illustration of this kind of navigation - i.e. of the basic form of gameplay on offer in Inglenook.

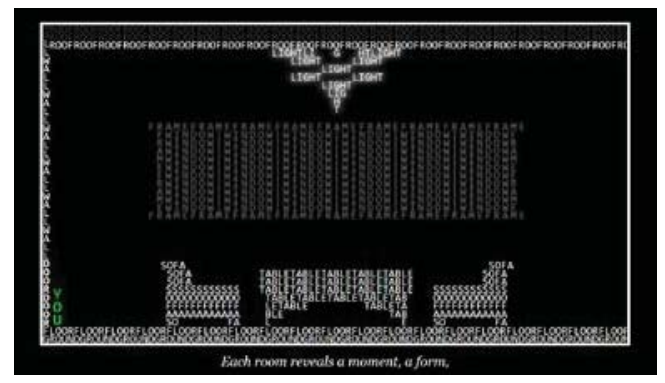

Figure 1. The initial passage of the living room space in Inglenook. The line of text on the bottom is the space's thematic text, appearing only during the first time this passage is visited.

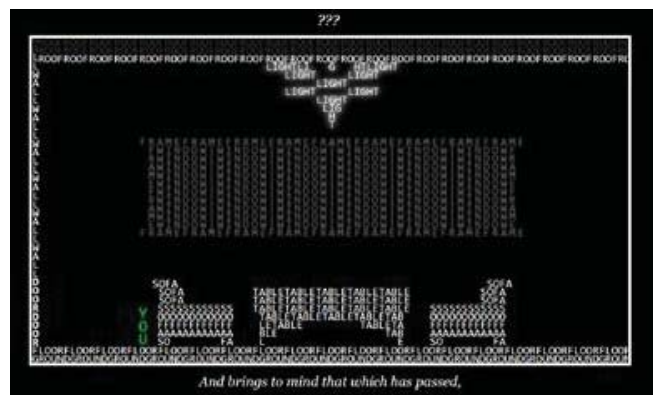

Figure 2. The subsequent living room passage, following a single movement/press of the right arrow key. Note the different position of the green word YOU. The question marks at the top indicate that the current position is an interaction point; the player can examine the sofa object by pressing the Space bar.

The gameplay design of Inglenook was influenced by interaction formats characteristic of adventure games and hypertext fiction. The former influence is evident in the game's spatial and mechanical design and overall goal structure. As part of playing Inglenook, the player navigates the house of words, consisting of three floors and nine separate rooms, interacts with objects such as doors and switches at interaction points designated by question marks, and solves several simple puzzles involving item retrieval and code input to unlock one of the two ending states of the game.

Each passage in Inglenook contains a single line of thematic text which appears only once, the first time a passage is visited, making the navigation between passages akin to flipping pages in a picture book. The lines do not contain any information relevant for progressing through the house; they appear at the bottom of the screen and take about half a second to appear once the passage is first visited. If the player who moves too quickly between several passages, these lines will not display at all for the passages which were speeded through. Taken together, the lines in each of the passages that comprise a discrete space, such as the living room, form short poems which deal with themes of isolation, loneliness, and regret. The ephemeral quality of the thematic lines was a deliberate design choice, indicated to the player at the beginning of the game. The intent was twofold: firstly, to enhance the aesthetic experience of the thematic text, and secondly, to enable a slower, more deliberate form of engagement, contrasting the adventure game genre mechanics, and consequently to gauge player reactions to these kinds of engagement.

\section{B. Participants}

A total of ten participants (six male, four female, ages 21-33) took part in the study. They were recruited online, via posts on social media groups, as well as through word-of-mouth snowball sampling [25, p. 237]. The resulting group of participants was relatively heterogenous in terms of their digital game preferences and experiences. Four of the participants were game design students, while one had a master's degree in the same field and was working for a game developer at the time of the study. On the whole, they had a great degree of experience with various kinds of games, although they reported preferences towards different game types and gaming platforms. The other five participants had non-gaming educations and degrees. Overall, they reported very specific gaming preferences and had lower degrees of gaming experience, with one of them not having played digital games at all in the past year.

All participants were informed of the general nature, but not the specific topic, of the research study. In an attempt to elicit more honest thoughts about the game, they were also told that Inglenook was designed by a third party. As part of the recruitment process, they signed dedicated consent forms, allowing for the use of their data for research purposes. The forms were approved by the ethics committee at the researcher's institution prior to participant recruitment. Participants were also issued gift cards as compensation for their participation.

\section{Data collection}

stories, but was omitted in this project in favor of keyboard input, which allowed for a more natural mapping of movement to the arrow keys.
${ }^{2}$ Mousetrap, a JavaScript library for handling keyboard input developed by
Craig Campbell, was used to facilitate navigation with key presses rather
than mouse clicks. The latter method of interaction is typical of Twine 
Participation in the study consisted of a preliminary questionnaire, recorded play session, post-play-session semistructured interview, and a brief optional questionnaire around a week after the play session.

The initial questionnaire contained questions on current and past gaming habits, type of participation in gaming culture, personal gaming history, game preferences and attitudes, as well as general media habits, preferences, and attitudes. The aim of the questionnaire was to capture multiple aspects of one's relationship with the field of gaming, as opposed to a single metric (e.g. preferences towards specific game types), as is the case in more dedicated questionnaires and psychometric scales. Because of the nature of its construction, the questionnaire does not serve as a tool for directly comparing one participant to the next. Rather, it acts as a rich source of background data for each individual participant, painting a picture of their own, unique relationship with the domain of digital games and other media. This approach to background data is in keeping both with the topic under investigation and the overall holistic aim of the study.

Due to restrictions on physical gatherings on the count of the coronavirus pandemic, the play sessions took place via Zoom. To facilitate this, the game prototype (in the form of an HTML file) was shared with the study participants at the start of the conversation. The testing session lasted between 30-50 minutes, with the participants playing the game for around ten minutes with their screen shared, and then being asked a series of questions as part of the post-playsession interview. To avoid being pressured for time, the participants were only told that they would be asked to stop playing after an arbitrary period. The Zoom session was recorded, and the interview portion later transcribed by the researcher.

Much like with the background questionnaire, the questions in the post-play-session interview were formulated as open-ended, to better capture the breadth of the participants' experiences with Inglenook and any other game and/or media product of which it had reminded them. The questions were subdivided into three groups: Gameplay Experience, Appreciation, and Comparisons. The first group of questions focused on the participants' opinions about the design of the game, both on a micro level (visuals, themes, mechanics, challenges) and on a macro level (impressions of the overall design approach behind the game). The second group of questions pertained to the appeal that Inglenook carried for the participants, and included questions on cultural perception and positioning of the game within the broader field of digital games and gaming. As part of the final group of questions, the participants were asked to compare Inglenook to other games that they may have played or heard of, as well as to other media products of which the game had reminded them during the play session.

At the end of the testing session, the participants were told that the game file was theirs to keep, and asked to participate in an optional follow-up questionnaire, which was distributed to them five to seven days following the testing session and which focused on their experiences with the game in this period. Only one of the participants did not take part in this portion of the study, and out of the remaining nine, three did not return to the game following the testing session or the time immediately after it. There were two reasons for conducting a follow-up questionnaire. Firstly, it was deemed important to allow participants time with the game outside the confines of the testing session, so that they would have a chance to play it on their own terms and complete it, if they so wished. Furthermore, since the participants had time to play the game of their own accord following the testing session, there was a chance that their opinions and feelings towards the game would change and evolve. In light of the overall goal of the study, this evolution was considered relevant and important to track.

\section{Data analysis}

Data obtained from the study encompassed around $6 \frac{1}{2}$ hours of video material and over 70 pages of interview transcripts, in addition to textual data and Likert scale responses from the two questionnaires. The transcripts were first prepared for analysis in the MAXQDA 2020 software package by using the open coding process [30]. Two rounds of open coding were conducted; the final refined code set included 40 different codes grouped into 13 main categories, and a total of 526 coded interview segments. The initial approach to the coded segments was focused on betweenparticipant comparison, in an attempt to determine and classify shared patterns of thought or opinion among the study participants. While this method did result in interesting observations about the participants' cultural perception of the gaming field and the prototype's position within it, it did not prove particularly fruitful in answering the questions behind the study.

As a result of this, a second round of analysis was performed, utilizing the same code system and segments, but instead examining the participants' responses individually and in depth, in light of their gaming experiences and attitudes reported in the initial questionnaire and their ingame behavior. In essence, this approach treated each participant in the study as an isolated case, seeking to determine - on the basis of interview data and gameplay recordings - how their own ludic habitus reacted when encountering Inglenook. Only after all participants' data were analyzed in this fashion was it possible to cross-examine them and draw conclusions regarding the study topic.

\section{RESULTS}

\section{A. Player clusters}

The participant profiles created in the second round of analysis were compared and contrasted, with participants with similar playstyles grouped together in a specific cluster. A total of three clusters were thus created:

1. Cluster One (C1; The Puzzle-Solvers) is comprised of three participants whose playstyles were fast-paced and who quickly disregarded the game's thematic text in favor of rapidly navigating the game's setting and solving the puzzles they encountered.

2. Cluster Two (C2; The Detectives) is comprised of two participants whose playstyles mainly revolved around investigating the game's mysterious setting 
and theme, with a moderate degree of engagement with the thematic text.

3. Cluster Three (C3; The Explorers) is comprised of five participants whose playstyles were the most methodical and slow-paced; they generally showed a high level of engagement with the virtual environment and with the thematic text, only skipping or missing a few of its lines.

The classification does not imply value judgments towards any of clusters nor any of the players, nor is it meant to flatten the individual differences between them. There were at times considerable differences between the participants belonging to the same cluster, though they were still grouped together on the basis of playstyle similarities. Instead, participant clustering is used to deliver a clearer, more organized presentation of the study data, which will help answer the research questions behind the study in a more systematic and comprehensive manner.

The three clusters are presented below. For the sake of relevance to the research questions, the summaries focus on matters of playstyle, opinions regarding thematic text and visual style, and cultural perception and positioning of Inglenook.

\section{1) Cluster One - The Puzzle-Solvers}

The first cluster consists of three participants: Arthur $^{3}$ (a PC gamer of European RPGs and grand strategy games), Joe (a PC and console gamer of Western RPGs, FPS games and fighting games), and Jill (a non-player, familiar with a handful of older, prominent gaming titles).

Despite differences in their level of practical familiarity with digital games, these three participants all played Inglenook in a similar fashion. The first several minutes of their playthroughs were characterized by slower, more exploratory engagement, which saw them investigating the ground floor of the house and turning on the lights upstairs. During this time, they moved relatively slowly between passages, only skipping a few lines of thematic text while navigating the ground floor. However, once these players solved the first puzzle in the game - which involves restoring power and unlocking access to the upper floors of the house by flipping a single switch on the ground floor they began playing at a more rapid pace, skipping through most of the lines of thematic text and orienting themselves firmly towards finding and solving the game's puzzles. Joe played the fastest of all three, and at times, his movements were so quick and erratic that they triggered visual glitches in the form of misaligned and misplaced text which made up certain objects. Of the three, Arthur (who played slightly longer than the other participants in the study, due to connection issues) progressed the furthest in the game, followed by Joe and Jill.

In the post-play-session interview, the three participants in this cluster expressed initial confusion, at times even annoyance, due to the game's visual and mechanical design, although they also praised the game's atmosphere, intensity of mood, and minimalism. Both Arthur and Jill described the game as a demo or an unfinished product, primarily because of the visual design. Out of all the participants in the study, Jill was most confused by the game; she mentioned that playing it felt like trying to exit an escape room. In the case of Joe, the game was an interesting concept, with simple gameplay mechanics that were just about adequate when paired with the unique visual style. Overall, thought, he felt that the game was too rough to appeal to him. Though all three participants mentioned not typically playing games like Inglenook, Joe was by far the most vocal of the three about his distant attitude towards the game. When asked if he considered Inglenook to be his kind of game, Joe replied:

\begin{abstract}
Joe: No. Absolutely not. Yeah (laughs), first because of the graphic design, which - if I want more of this kind of involved thinking, I would choose reading a book or, I dunno, participating in a discussion and not to go into a computer game, from which I expect more of a relatively easy entertainment.
\end{abstract}

The three participants in $\mathrm{C} 1$ also shared similar reservations towards the thematic text. All mentioned reading it at first, but then, at some point, realizing it is not relevant for the gameplay, and subsequently skipping it in favor of puzzle-solving. All three stated that the text contributed to the atmosphere and feel of the game, but ultimately, puzzlesolving proved to be more of a draw for them as players. This can also be seen in their gameplay recordings. As mentioned before, once these players solved the first puzzle and unlocked the upstairs area of the house, their engagement with the game changed from being relatively balanced between reading and exploration, to predominately goaloriented. At that point, for all three players, Inglenook seemed to become fixed as an adventure-puzzle game, rather than a hybrid of digital game and hypertext fiction.

\section{2) Cluster Two - The Detectives}

The second cluster consists of two participants: Willow (smartphone and PC player of story-based games, point-and-click adventures, and city building games) and Alice (a multiplatform player of interactive visual novels, story-based games, and puzzle/adventure games). Both are students of game design, who regularly make games and attend game jams, with Willow also creating fan art and Alice working on visual art in general.

Much like the other participants, Willow and Alice at first played slowly, reading each of the lines of thematic text as they would appear on screen. However, as they were moving from room to room on the ground floor, their engagement with the game coalesced into an investigative playstyle, with quicker navigation between passages and more time spent interacting with points of interest in the various rooms. Unlike the first group, this did not mean that Willow and Alice began to disregard the thematic text altogether. Rather, it seemed as though they were now predominately playing Inglenook as an adventure game that had an underlying mystery. Their primary goal was to investigate the space of the house - for example, by sequentially interacting with all objects in a room and moving quickly towards switches whenever they would see them. Throughout their playthroughs, this investigative style of play seemed to also make room for reading the snippets of 
text at the bottom of the screen. Alice seemed to be the more diligent reader, skipping fewer lines overall. Willow, on the other hand, would occasionally move too quickly between two passages, especially when entering a new room, and would then try and go back in order to read the skipped text.

In the post-play-session interview, Willow admitted to not reading more than half of the thematic text, despite wanting to do so, on the count of excitement brought about by investigating the house. Nevertheless, both her and Alice agreed that the thematic text contributed to the game feeling like a mystery waiting to be solved, and that it worked well in tandem with the space, gameplay, and visual style. Both players remarked on Inglenook's hybrid nature as both a puzzle-based adventure game and a work of hypertext fiction:

Willow: [...] I feel like it was adding a lot to the story where you had this really, like, minimalistic thing going on. So it kind of felt together, like you were playing more of a book than a game, which was really fun. I feel like it added a lot.

Alice: I think that was the part that made the game mysterious. Like, the way that the text was written [...] That was where I got the mysterious feeling the most. And it was... It also seemed like, like a book, or something like that, that you are going to read through the rooms of the home.

The visual style elicited somewhat different responses from the two participants in this cluster. Willow was very enthusiastic about it, even going as far as describing the game as artistic and experimental due to its visuals and comparing it to works which challenge one's conception of a given medium. She also considered the game well-designed - for "an indie game [and] a short project made by one person." Alice was more reserved in her evaluation. For her, the visuals were interesting and nice, but they were also a mark of the game's incomplete status. On more than a single occasion, she mentioned the visual style of Inglenook being "preferable to bad art." Even though Willow and Alice saw the game in a different light - as an artistic experiment and an unfinished prototype, respectively - they both seem to be more aware of its dual identity as a digital game/interactive fiction hybrid. For the two of them, Inglenook primarily seemed to be a digital space inviting investigation, one in which they could solve puzzles and also follow a sort of story.

\section{3) Cluster Three - The Explorers}

The third, final, and biggest cluster consists of the remaining five participants: Peter (a PC player of open-world games), Susan (a PC player of FPS games), Thomas (a PC player of FPS, strategy, and action-adventure narrative games), Evan (a console player of platformers, actionadventure, and puzzle games), and Miles (a PC and console player of FPS games, crafting games, and indie games with unique mechanics). The latter three were or are game design students and makers of digital games.

All five of the players in this cluster played Inglenook more slowly and methodically than the other participants in the study. From beginning to end of their play experience, they played in a manner which indicated they were reading the thematic text, skipping these lines only on rare occasions or by accident. In this cluster, gameplay speed did not seem to be affected by time already spent playing, or by reaching a specific point in the game, as was the case in the other two clusters. These five players were not just playing slowly because they had trouble with the controls or navigating the virtual environment: they were perfectly capable of speeding up when navigating already familiar rooms. Rather, their attention seemed to be relatively balanced between reading the thematic text, exploring the virtual environment, and solving the game's puzzles.

The interviews after the play sessions revealed a high degree of appreciation for the game's visual style, but differing attitudes towards the game's thematic text. Peter and Evan considered the text confusing and at times annoying in its presentation, admitting that their appreciation and focus towards it waned as the game progressed. Despite this, they kept on trying to read the lines:

Peter: The only thing which was annoying for me was that I had
to wait for every single step, so I [could] read - and I skipped a
lot of [the text], because, by habit, when you play, you're not
supposed to just tap-wait-tap-wait... And I was just, like, skipping
those [lines], but not intentionally. Sometimes I even went back
to see if I can read it again.
Evan: I felt like the small text at the bottom stopped mattering to
me. [... B But I'm, like, the kind of player that get[s] a bit annoyed
by that, because I really want to take in everything. And then
when there's something that I don't find interesting, I force myself
to take it in, even though I'm not enjoying it a lot (laughs).

Susan, Thomas, and Miles, on the other hand, were more enthusiastic about the text, even though they also found it confusing at times. For them, the thematic text felt like a very important and relevant component of the game's design, enriching their experience and letting their minds wander as they played. Susan mentioned that the lines of thematic text "gave the game itself some [...] definition [and] depth." For the three players with game design backgrounds - Miles, Thomas, as well as Evan - the thematic text (coupled with the game's visual style and other design elements) led to a categorization of Inglenook as an independent or indie game, drawing comparisons with titles such as What Remains of Edith Finch and Limbo. In the case of Miles and Thomas, this perception of Inglenook was enough for them to adopt a very specific stance towards it. This stance influenced, among other things, their degree of engagement with the thematic text, as illustrated by Miles's comment below:

\begin{abstract}
Miles: If it's a game like this, where you're kind of, like, put in a narrative, I feel like I really want to read it. I feel like it is a very essential part of the story. But in other games, [...] like an Assassin's Creed game or something, where they put in audio longs or whatever, I don't feel like I want to read it, because I don't feel like it adds anything to it.
\end{abstract}

Despite differences in level of appreciation for the thematic text, the five players in this cluster approached Inglenook on similar terms. As seen from their gameplay footage and interview responses, the main allure of the game for them did not seem to lie exclusively in any individual aspect of the game's design, such as puzzles, space, or story. Rather, each of these players, in turn, tried to explore and experience as much of the game and its various aspects as possible throughout their time with Inglenook. This resulted in a slower, more comprehensive form of engagement than that of participants in either of the two other clusters. For the five participants in C3, Inglenook never coalesced solely into a puzzle game or an adventure game or a work of interactive fiction, but rather retained the properties of all of these forms during the entirety of their respective play sessions. 


\section{DISCUSSION}

The results show that participants in the study played Inglenook in one of three different styles - with a focus on solving puzzles $(\mathrm{C} 1)$, investigating the house $(\mathrm{C} 2)$, or in a more balanced, slower fashion which encompassed puzzle-solving, exploration, and thematic text reading (C3). Now we can return to the questions which motivated the study, and ask them again, in light of these results. How did these three playstyles emerge? What factors were involved in bringing them to light?

To answer these questions, we need to take a closer look at the behavior of the study participants. All three groups of players seem to take cues from specific elements of the game's design, which induced a specific style of play:

- For C1 players, the playstyle change happened when they solved the first puzzle in the game - i.e., when they restored power to the upper floors of the house. The presence of puzzles in the game was, on its own, enough to cue them into a more rapid, problemsolving playstyle.

- For C2 players, the playstyle change seemed to take place when investigating rooms on the ground floor. The mysterious atmosphere in the game, generated by a combination of design elements (unorthodox visual style, poetic thematic text, and abandoned house setting, among others) cued these players into becoming quick, meticulous detectives.

- For C3 players, no notable change in playstyle took place during their time with the game. However, all five players in this cluster were influenced in their style of play by the thematic text at the bottom of the screen. This engagement meant that they maintained a steady, relatively slow style of play, alternating between reading the text, navigating the virtual environment, interacting with objects, and solving puzzles.

At first glance, the differences between the groups might also be attributed to their level of domain knowledge i.e. play and cultural experience with certain subfields of gaming practice. Players in $\mathrm{C} 1$ had limited experience with games that share Inglenook's mechanics, visual perspective and layout, or aesthetic experimentation - or just limited experience with digital games in general. Conversely, three out of five of the players in $\mathrm{C} 3$ are or were game design students, with an extensive knowledge of various game genres and genre conventions, including indie/art/experimental games. What is more, they did not hesitate labeling Inglenook as just such a game, which, as Miles pointed out, did influence their mode of engagement with it. It is tempting to say that the ludic habitus of $\mathrm{C} 1$ players were underdeveloped, or simply not attuned to the subfield of narrative indie games such as Inglenook, and that the ludic habitus of $\mathrm{C} 3$ players matched the requirements and conventions of the game. The data, however, paints a more complex picture: $\mathrm{C} 3$ also had two players who were not wellversed in indie or experimental games, and both players in $\mathrm{C} 2$ were also game design practitioners and quite familiar with experimental game titles, yet played in a different way to $\mathrm{C} 3$ players. Within the framework of ludic habitus and subfields of practice, how are we to explain these peculiarities?
To an extent, possessing domain knowledge and practical experience with a subfield of practice such as indie/experimental games did seem to translate into greater appreciation, deeper and richer analysis, and a more comprehensive engagement with the various components of the game's design (principally, thematic text and visual style). Beyond that, it would seem that personal play preferences played a major role in determining how a particular player would navigate Inglenook. Put simply: when caught between the drive to solve puzzles, explore a large, mysterious home, and/or read poetic text, players simply played the way that suited them, engaging with those elements that mattered the most to them as players.

Among the participants, these preferences seemed to be linked to several different factors, and to either be marks of their general attitudes to games or very context- or subfield-dependent. As an example, let us look at the participants' engagement with the thematic text. Regardless of the degree to which they read it as individuals, the players in $\mathrm{C} 3 \mathrm{read}$ the thematic text the most of the three clusters. They did so either because they feel compelled to read everything in games (Evan), enjoy reading narrative in games (Peter), enjoyed the thematic text in this game (Susan), or because they recognized the text as relevant and meaningful in light of the game's overall experimental/artistic character (Thomas, Miles). For the two players in $\mathrm{C} 2$, the text was not as much of a draw as the other elements of the game's design (gameworld, puzzles, visual style), and the excitement of exploration (Willow) or solving a mystery (Alice) ultimately prevailed and influenced their style of play. The players in $\mathrm{C} 1$ did not care much for the thematic text, focusing exclusively on the puzzles and completing the game, either because that is how they usually play games (Joe, Arthur), or because that is what they thought this game was about (Jill).

\section{LIMITATIONS AND DIRECTIONS FOR FURTHER RESEARCH}

The study was conducted using a custom-made game prototype; none of the study participants had played it prior to their respective Zoom sessions, which were capped at around ten minutes of playtime. Prior familiarity with a given game, as well as the freedom to play it at one's own pace, will almost certainly impact one's playstyle. This was evident from the follow-up questionnaire responses, where, for example, C2 players (Willow and Alice) reported playing more slowly and with more time devoted to the thematic text in the period after the Zoom session. In light of this, as well as the limited number of study participants, the study findings should best be understood as hypotheses, pertaining only to first instances of playing a particular digital game. Longer observational studies with more participants would be needed to further explore how one's ludic habitus impacts one's engagement with different aspects of a digital game over time and with repeated plays. Additional exploratory studies examining other facets of ludic habitus would also contribute to a more detailed understanding of the concept and how it applies to digital - and other types of - gaming.

The fact that Inglenook is a single-player digital game must also be taken into account when discussing the study results. Though it was beyond the scope of this study to investigate it, it seems reasonable to claim that the presence and behavior of other players would be considerable factors 
influencing how one engages with a digital game, novel or familiar. Further research with multiplayer games could shed important light on interactions not just between game design and one's ludic habitus, but also between ludic habitus of various players in the same game setting.

\section{CONCLUSION}

The study results show that two main factors influence a player's style when playing a new digital game.

1) Firstly, elements of a game's design - e.g. its visual style, gameplay mechanics, ludic systems, and narrative content - cue specific interpretations and afford specific forms of engagement to the player.

2) Secondly, the player's ludic habitus - the collection of their game-domain-related experiences, knowledges, and attitudes - interprets the game's design both continuously (as part of the moment-to-moment interaction with the game system) and contextually (by comparing each aspect of the game's design with others, in the context of the game as a whole, and with previously encountered games).

As examples from $\mathrm{C} 1$ and $\mathrm{C} 2$ show, playstyles often consolidate at specific moments of discovery - i.e., when certain elements of a game's design cue the player into a breakthrough or realization about the nature of the game they are playing, which, in turn, leads them to adopting a specific stance towards the game as a whole. These moments of discovery are often contingent on one's familiarity with games of a similar genre or type - that is, by other games belonging to the given subfield of practice. While it seems that the most relevant arbiters of choice between modes of engagement are personal preferences for particular ways of playing, these preferences are often bound to design conventions in a given subfield of practice. In other words, players assign relevance to certain aspects of a game's design (mechanics, narrative, visual style, etc.) by viewing them in different contexts: of the game as a whole, of other games they have played, and of the subfield of practice (if any) to which they think it belongs. In the end, the player's interpretation of the game as a specific kind of game, emerging from their individual analysis of its design, sets the stage for how they will engage with it.

\section{REFERENCES}

[1] T. H. Apperley, "Genre and game studies: Toward a critical approach to video game genres," Simulation \& Gaming, vol. 37, no. 1, pp. 6-23, 2006.

[2] T. Apperley and C. Walsh, "What digital games and literacy have in common: a heuristic for understanding pupils' gaming literacy," Literacy, vol. 46, no. 3, pp. 115-122, 2012.

[3] D. Arsenault, "Video game genre, evolution and innovation," Eludamos: Journal for Computer Game Culture, vol. 3, no. 2, 2009.

[4] R. Bartle, "Hearts, clubs, diamonds, spades: Players who suit MUDs," Journal of MUD research, vol. 1, no. 1, 1996.

[5] C. Bateman, R. Lowenhaupt and L. E. Nacke, "Player typology in theory and practice," in DiGRA Conference: Think Design Play, 2011, pp. 1-24.

[6] M. Bialas, S. Tekofsky and P. Spronck, "Cultural influences on play style," in 2014 IEEE Conference on Computational Intelligence and Games, 2014, pp. 1-7.

[7] B. Bostan, "Player motivations," Computers in Entertainment, vol. 7, no. $2,2009$.

[8] P. Bourdieu, "Social space and symbolic power," Sociological Theory, vol. 7, no. 1, pp. 14-25, 1989.

[9] P. Bourdieu, In other words: Essays towards a reflexive sociology, Stanford, CA: Stanford University Press, 1990.
[10] P. Bourdieu, Outline of a theory of practice, Cambridge: Cambridge University Press, 1972/2013.

[11] D. Carr, "Contexts, gaming pleasures, and gendered preferences," Simulation \& Gaming, vol. 36, no. 4, pp. 464-482, 2005.

[12] T. Cole and M. Gillies, "Thinking and Doing: Challenge, Agency, and the Eudaimonic Experience in Video Games," Games and Culture, vol. 16, no. 2, pp. 187-207, 2021.

[13] M. Consalvo, Cheating: Gaining Advantage in Videogames, Cambridge, MA: MIT Press, 2007.

[14] D. R. Dietrich, "Avatars of whiteness: Racial expression in video game characters," Sociological Inquiry, vol. 83, no. 1, pp. 82-105, 2013.

[15] A. Drachen, A. Canossa and G. N. Yannakakis, "Player modeling using self-organization in Tomb Raider: Underworld," in 2009 IEEE Symposium on Computational Intelligence and Games, Milan, Italy, 2009 , pp. $1-8$

[16] A. Drachen, R. Sifa, C. Bauckhage and C. Thurau, "Guns, swords and data: Clustering of player behavior in computer games in the wild," in 2012 IEEE Conference on Computational Intelligence and Games (CIG), Granada, Spain, 2012, pp. 163-170.

[17] J. Hamari and J. Tuunanen, "Player Types: A Meta-synthesis," Transactions of the Digital Games Research Association, vol. 1, no. 2, 2014.

[18] C. Heeter and B. Winn, "Gender identity, play style, and the design of games for classroom learning," in Beyond Barbie and Mortal Kombat: New perspectives on gender and gaming, Y. Kafai, C. Heeter, J. Denner and J. Sun, Eds., Cambridge, MA: MIT Press, 2008, pp. 281300.

[19] B. Ip and G. Jacobs, "Segmentation of the games market using multivariate analysis," Journal of Targeting, Measurement and Analysis for Marketing, vol. 13, no. 3, pp. 275-287, 2005.

[20] J. Jenson and S. de Castell, "Girls@ Play: An ethnographic study of gender and digital gameplay," Feminist Media Studies, vol. 11, no. 2, pp. 167-179, 2011.

[21] K. P. Kallio, F. Mäyrä and K. Kaipainen, "At least nine ways to play: Approaching gamer mentalities," Games and Culture, vol. 6, no. 4, pp. 327-353, 2011.

[22] G. Kirkpatrick, The formation of gaming culture: UK gaming magazines, 1981-1995. Basingstoke: Palgrave Macmillan, 2015.

[23] R. Klevjer and J. F. Hovden, "The structure of videogame preference," Game Studies, vol. 17, no. 2, 2017.

[24] W. McNeish, and S. De Paoli, "Developing the Developers: Education, Creativity and the Gaming Habitus," in Proceedings of the 1st International Joint Conference of DiGRA and FDG, Dundee, Scotland, 2016, pp. 1-2.

[25] M. Q. Patton, Qualitative Research and Evaluation Methods, Thousand Oaks: SAGE, 2002.

[26] C. Pearce, "The Truth About Baby Boomer Gamers," Games and Culture, vol. 3, no. 2, pp. 142-174, 2008.

[27] F. Raczkowski, “'And what do you play?"”: A few considerations concerning a genre theory of games," in Computer Games and New Media Cultures, Dordrecht: Springer, pp. 61-74, 2012.

[28] R. M. Ryan, C. S. Rigby and A. Przybylski, "The motivational pull of video games: A self-determination theory approach," Motivation and emotion, vol. 30, no. 4, pp. 344-360, 2006.

[29] T. Sihvonen and J. Stenros, "Cues for queer play: Carving a possibility space for LGBTQ role-play," in Queerness in Play, Palgrave Macmillan, pp. 167-184, 2018.

[30] A. Strauss and J. Corbin, Basics of qualitative research: Techniques and procedures for developing grounded theory. Thousand Oaks, CA: Sage, 1998.

[31] S. Tekofsky, P. Spronck, M. Goudbeek, A. Plaat and J. van den Herik, "Past Our Prime: A Study of Age and Play Style Development in Battlefield 3," in IEEE Transactions on Computational Intelligence and AI in Games, vol. 7, no. 3, pp. 292-303, Sept. 2015

[32] J. Vahlo, J. K. Kaakinen, S. K. Holm and A. Koponen, "Digital game dynamics preferences and player types," Journal of ComputerMediated Communication, vol. 22, no. 2, pp. 88-103, 2017.

[33] M. J. P. Wolf, The medium of the video game. Austin, TX: University of Texas Press, 2001.

[34] N. Yee, "Motivations for play in online games," CyberPsychology \& Behavior, vol. 9, no. 6, pp. 772-775, 2006

[35] F. Zhu, "Computer gameplay and the aesthetic practices of the self: Game studies and the late work of Michel Foucault," Transactions of the Digital Games Research Association, vol. 3, no. 3, pp. 75-104, 2018. 